\title{
A new approach to quantify internal and external training load for intermittent sports
}

\author{
Un nuevo abordaje para cuantificar carga interna y externa de entrenamiento \\ en deportes intermitentes
}

\begin{abstract}
Roberto Andrés González-Fimbres*, María Grethel Ramírez-Siqueiros', Luis Felipe Reynoso-Sánchez², José Trinidad Quezada-Chacón ${ }^{3}$, Janeth Miranda-Mendoza ${ }^{4}$ y Germán Hernández-Cruz ${ }^{4}$

Universidad Estatal de Sonora. Ley Federal del Trabajo S/N, Colonia Apolo, Hermosillo, Sonora, México, CP 83100.

2 Universidad Autónoma de Occidente, Dpto. Ciencias Sociales y Humanidades. Blvd. Macario Gaxiola y Carretera Internacional, s/n. Narciso Mendoza, Los Mochis, Sinaloa, México. C.P. 81217.

${ }^{3}$ Universidad Autónoma de Ciudad Juárez. Instituto de Ciencias Biomedicas. Av. Benjamin Franklin no. 4650 Zona PRONAF CP 32315.

Universidad Autónoma de Nuevo León, Facultad de Organización Deportiva. Cd. Universitaria, S/N, San Nicolás de los Garza, Nuevo León, México, C.P. 66455.
\end{abstract}

\section{ABSTRACT}

The aim of this study was to propose a modified training impulse method (TRIMP) to quantify internal training load (ITL) in intermittent team sports and examine its relationship with external training load (ETL) during a preparatory period. Over 12 weeks, 11 male youth field hockey players (14.41 \pm 0.51 years) were evaluated in regard to their ETL using triaxial accelerometers (Actigraph) and data was later contrasted with ITL, which was measured using heart rate (HR) monitors (Polar Team2) by four different TRIMP methods: Banister's (bTRIMP), Edwards's (eTRIMP), individualized (iTRIMP) and modified (mTRIMP). A correlation was found between HR (beat $/ \mathrm{min})$ and ETL $\left(r=0.699, \mathrm{R}^{2}=0.489, p\right.$ $<0.01)$ and among TRIMP methods $(r=0.808-0.984, p<0.01)$, however, the consistency between methods did not agree ( $p$ $<0.01)$. The ETL correlated in all TRIMP methods: bTRIMP ( $r=$ $\left.0.509, \mathrm{R}^{2}=0.259, p<0.01\right)$, eTRIMP $\left(r=0.336, \mathrm{R}^{2}=0.113, p<\right.$ $0.01)$, iTRIMP $\left(r=0.224, R^{2}=0.050, p<0.01\right)$ and $\operatorname{mTRIMP}(r=$ $\left.0.516, \mathrm{R}^{2}=0.267, p<0.01\right)$. The proposed mTRIMP can be a valid option for ITL quantification; furthermore, indexes combining ITL and ETL should be used for a complete training assessment.

Keywords: intermittent exercise; TRIMP; accelerometer; field hockey.

\section{RESUMEN}

El objetivo del estudio fue proponer un método modificado de estímulo de entrenamiento (training impulse, TRIMP) para cuantificar carga interna (Cl) de entrenamiento en deportes de equipo intermitentes y examinar su relación con la carga externa (CE) durante un periodo de preparación. Durante 12 semanas, se evaluó la CE de 11 jugadores de un equipo juvenil varonil (14.41 \pm 0.51 años) de hockey sobre pasto utilizando acelerómetros triaxiales (Actigraph) y la $\mathrm{Cl}$ con monitores (Polar Team2) de frecuencia cardiaca (FC) por medio de cuatro diferentes métodos de TRIMP: Banister (bTRIMP), Edwards (eTRIMP), Individualizado (iTRIMP) y Modificado (mTRIMP). Se encontró relación entre FC y CE ( $r=.699$,
$\left.\mathrm{R}^{2}=0.489, p<0.01\right)$ y entre los cuatro métodos de TRIMP $(r$ $=.808-.984, p<0.01)$, aunque no hubo concordancia entre ellos $(p<0.01)$. La CE tuvo correlación con bTRIMP $\left(r=.509, R^{2}\right.$ $=.259, \mathrm{p}<0.01)$, ETRIMP $(r=.336, \mathrm{R} 2=.113, p<0.01)$, iTRIMP $\left(r=224, \mathrm{R}^{2}=.050, p<0.01\right)$ y mTRIMP $\left(r=.516, \mathrm{R}^{2}=.267, \mathrm{p}<\right.$ $0.01)$. El mTRIMP puede ser una opción válida para cuantificar $\mathrm{Cl}$, además, se deben utilizar índices de $\mathrm{Cl}$ y la CE para una valoración global del entrenamiento.

Palabras clave: Ejercicio intermitente; TRIMP; acelerómetro; hockey sobre pasto.

\section{INTRODUCTION}

Periodization is a process where coaches alternate load and recovery phases to improve athletes performance (Turner, 2011; Deweese et al., 2015). The amount of exercise exerted by the athletes (distance, power output, number of repetitions), with no regard of internal effects, is known as external training load (ETL); the physiological response to ETL (oxygen uptake, heart rate, blood lactate, rate of perceived exertion) is considered internal training load (ITL) (Buchheit, 2014). Precise and reliable ITL and ETL quantifying methods are required to analyze and establish causal relationships between training, physiological adaptations, and resulting performance level (Mujika, 2013; Halson, 2014). During team sports matches and training sessions players execute sport specific actions at diverse intensities, interjecting loading and resting periods in unpredictably way, which imposes players unique physiological demands (Vinson \& Peters, 2016). Since team sports activity is intermittent by nature, it is difficult to quantify ETL and ITL, which is the reason for the need of individual quantification.

For ETL quantification in team sports, global positioning system (GPS) movement tracking devices have been used (Jennings et al., 2012; Suarez-Arrones et al., 2012; Varley et al., 2012) as well as video-based time motion analysis (TMA) after matches or training sessions (Abdelkrim et al., 2007; Klusemann et al., 2013; Canovas et al., 2014). Nevertheless, given the imprecision of GPS measurements (Varley et al.,

*Autor para correspondencia: Roberto Andrés González-Fimbres Correo electrónico: roberto.gonzalez@ues.mx

Recibido: 4 de julio de 2018

Aceptado: 4 de noviembre de 2018 
2012) and that TMA is time consuming (Canovas et al., 2014), the use of both methods present significant disadvantages (Scanlan et al., 2014). Recently, the use of accelerometers for ETL quantification in team sports has been supported (Scanlan et al., 2014), given that it overcomes TMA and GPS limitations (Scott et al., 2012; Casamichana et al., 2013; Scott et al., 2013; Scanlan et al., 2014).

According to fitness-fatigue paradigm (Morton et al., 1990), ITL can be quantified by heart rate (HR) based TRIMP methods. Banister (1991) proposed the first TRIMP (bTRIMP), which considers exercise intensity as heart rate reserve (HRres) and exercise volume as duration in minutes. Mean HRres multiplied by training session minutes, and by a weighting factor, represents the relationship between fractional elevation of blood lactate (Bla) and HRres during an incremental test. The utilization of bTRIMP for ITL quantification for intermittent team sports has been questioned given the notion that mean HRres does not accurately reflects HR fluctuations during training sessions (Alexiou \& Coutts, 2008). For this reason, bTRIMP has been adapted for ITL quantification at intermittent team sports. Edwards (1994) proposed a TRIMP modification (eTRIMP) classifying HR values at five zones of percentage of HR maximum (\%HRmax), then the sum of minutes spent at each zone is multiplied by an arbitrary weighting factor. The use of training zones is considered a limitation for eTRIMP, as it ponders time spent at upper and lower zone limits as equal; another limitation is the lack of physiological basis for each zone's weighting factors. To overcome these limitations, Manzi (2009) proposed an individualized TRIMP (iTRIMP). This method assesses the individual Bla responses to effort taking into account every single HR value observed during the training session (Manzi et al., 2010). While evidences indicates that iTRIMP has a high doseresponse relationship at intermittent sports training, this method requires submitting players to maximal incremental tests to obtain Bla profiles, which represents difficulties as invasiveness, high economical costs, access to lab facilities, specialized equipment, and trained personnel.

Since it was observed that different individual ETL at the same group of athletes on any given session, and at the same time, experience different ITL depending on conditioning, background, and genetic characteristics (Lambert \& Borresen, 2010), coaches should not design training programs based just in ITL or ETL, since both of them contribute to a total athlete's TL quantification. A combination of both methods appears to be key for appropriate monitoring (Halson, 2014). To our knowledge, no studies that propose objective parameters of ITL and ETL ratios are available. For this reason, this study aimed to propose a new modified TRIMP for ITL quantification at intermittent team sports, which avoids the use of training zones and uses generic weighting factor and examine its relationship with other TRIMP methods and ETL during a training period. We hypothesized that this novel mTRIMP method accurately represent ETL in intermittent training and have construct validity with other well stablished TRIMP methods.

\section{MATERIALS AND METHODS Design}

This study had a quantitative approach with a descriptive and correlational scope. Design was observational non-experimental.

\section{Sample}

A non-probabilistic, sampling by convenience, single group method was employed. Eleven male Sub-16 field hockey players took part in this study; they were members of the Mexican national champion team (Demographic info is presented in Table 1). Participants were all volunteers. Since all subjects were underage, we obtained a written informed consent from their parents or legal guardians. Study protocol followed the guidelines expressed by the Helsinki declaration and was approved by UANL's (Universidad Autónoma de Nuevo León) Health Sciences Research Bioethics Committee, No: COBICIS-58/12/2017/02-FOD-BRRC.

\section{Procedure}

Demographic info. Height (Digital stadiometer, model 274, Seca, Hamburg, Germany), weight, four-compartment body mass percentages (Medical digital scales, Model TBF_310, Tanita Corporation, Tokyo, Japan) and elbow and knee bone diameters (FUTREX caliper, Filderstadt, Germany) were measured. Before the start of protocol subjects underwent an intermittent fitness test (IFT 30-15), as specified by Buchheit (2010).

Incremental test. Bla profiles were determined for every subject with an incremental test. Protocol comprises successive stages of 3 minutes of treadmill run and a 1-minute passive recovery. Initial speed was set at $6 \mathrm{Km} / \mathrm{h}$, with $2 \mathrm{Km} / \mathrm{h}$ increments at the end of every stage until volitional fatigue (Manzi et al., 2009). Maximum HR value observed during the test was considered as HRmax. In the 1-minute interval between bouts, HR was recorded, and capillary blood samples were taken for Bla analysis (Accutrend Plus Lactometer, Roche Diagnostics, Mannheim, Germany). HR was recorded using Polar Team2 HR monitor system (Polar electro Oy, Kempele, Finland), every stage $H R$ value was determined as mean HR for every 3 minutes period.

Training sessions. Data recollection was made during a field hockey training program at the special preparation phase over a three-month period. Researchers did not have input on training session contents (designed and conducted by

Table 1. Subject's demographic info, mean and standard deviation.

Tabla 1. Media y desviación estándar de la información demográfica de la muestra.

\begin{tabular}{lcccccc}
\hline Age & Height & Weight & Fat $\%$ & Bone $\%$ & Muscle \% & VO2max \\
\hline $14.41 \pm 0.51$ & $168.23 \pm 5.25$ & $55.55 \pm 4.26$ & $11.49 \pm 2.93$ & $20.69 \pm 1.92$ & $46.81 \pm 3.22$ & $49.26 \pm 2.19$ \\
\hline
\end{tabular}


the coaching staff only). Training sessions were two hours long, with five days a week frequency. During the study every player participated in an average of 29.9 sessions; a total of 329 sessions were analyzed.

HR monitoring. Polar Team2 Pro HR monitors (Polar electro Oy, Kempele, Finland) were placed on each player during all training sessions. Transmitters were connected to an elastic chest strap, which had electrodes that has to be moisten for optimal skin contact. Sampling rate was at 1-second interval; each player's HR responses was recorded and exported to a personal computer for future analysis.

External training load. ETL was determined using triaxial accelerometers (Actigraph) placed at the back as instructed by Scanlan (2014). The devise was fixed onto the HR monitor chest strap using Velcro. This position places the accelerometer nearest possible to subjects center of mass, which better represents whole body movements. Each accelerometer had a full-scale output range of $\pm 6 \mathrm{~g}$ and sampled at a rate of $100 \mathrm{~Hz}$. Whole-body movements were determined as the accumulated instantaneous rate of change in acceleration in the three movement planes. ETL was then calculated using Player's Load methodology by the following formula:

$$
\text { Player's Load }=\sqrt{\frac{\left(a_{y 1}-a_{y-1}\right)^{2}+\left(a_{x 1}-a_{x-1}\right)^{2}+\left(a_{z 1}-a_{z-1}\right)^{2}}{100}}
$$

Where: $\mathrm{a}_{\mathrm{y}}=$ anteroposterior acceleration; $\mathrm{a}_{\mathrm{x}}=$ mediolateral acceleration; $\mathrm{a}_{\mathrm{z}}=$ craniocaudal acceleration. Accelerometer data at Player's Load arbitrary units of each training session were recorded in the Actigraph device. Subsequently, data was exported to a computer using Actilife software (version 6.13.3, 2016, Pensacola, Florida, USA). bTRIMP is calculated by multiplying mean HRres by session's total minutes and then by a weighting factor (Banister, 1991). HRres is calculated by the following formula:

$$
H R_{\text {res }}=\frac{H R_{\text {exe }}-F C_{\text {rest }}}{F C_{\text {max }}-F C_{\text {rest }}}
$$

Where: HRrest $=$ Heart rate at rest; HRexe $=$ Average heart rate during exercise; HRmax = Maximal heart rate. Session's bTRIMP is then calculated in arbitrary units using the following formula

$$
\operatorname{TRIMP}(A U)=H R_{\text {res }} * t * y
$$

Where: $\mathrm{t}=$ time in minutes, $\mathrm{y}=0.64^{* \mathrm{e} 1.92^{*} \text { Hres }}$ (for male subjects), e $=$ Neperian natural logarithm (2.718281828). eTRIMP. Edward's (1994) ITL quantification method intensity is weighted according to five zones relative to HRmax. Time in minutes spent at each zone is multiplied by an arbitrary weighting factor. At this study, eTRIMP was automatically calculated by Polar Team2 Pro software.

ITRIMP. Individual weighting factors were calculated using the exponential curve equation that better represents individual Bla response to incremental test. The equation consider " $y$ " value as Bla and " $x$ " value as HRres:

$$
y=a * e^{b * x}
$$

Once individual weighting factors are obtained, session iTRIMP is calculated with the sum of each observed by the following formula:

$$
\text { iTRIMP }=\sum H R_{\text {res }} * t * y
$$

Where: HRres $=$ Percentage of heart rate reserve observed at every 1 -second interval, $\mathrm{t}=$ time in minutes (at 1 -second interval $\mathrm{t}=1 / 60), \mathrm{y}=$ individual weighting factor.

mTRIMP. Calculation for mTRIMP was made similar to iTRIMP, with the only difference that instead of individual weighting factors, Banister's (1991) generic weighting factor is used by the following formula:

$$
m T R I M P=\sum H R_{r e s} * t *\left(0.64 * e^{1.92 * H R r e s}\right)
$$

Training ratios. To assess individual players' ITL to ETL relationships, several ratios were calculated. For ETL/time analysis Player's load:minute ratio was calculated. For ITL/ time analysis TRIMP:minute ratios were calculated by the four TRIMP methods. To analyze the internal effect of ETL TRIMP:Player's load ratios were also calculated by the four TRIMP methods.

\section{Statistical analyses}

All statistical analyses were conducted with SPSS statistical software (version 22 for Windows, SPSS Inc., Chicago, USA). Results are expressed as mean \pm SD. Data normality was proven with Shapiro-Wilk test. Pearson coefficient was used to examine correlation between HR and ETL, between TRIMP methods, and between TRIMP methods and player's load. Determination coefficient $\left(R^{2}\right)$ was used for correlation effect size evaluation, 0.8 and above was considered large, around 0.5 moderate and 0.2 or less as small (Manzi et al., 2009; Scanlan et al., 2014). To establish differences for Player's load, bTRIMP, ETRIMP, iTRIMP and mTRIMP between players a one way ANOVA analysis was used. ETA value was considered for effect size. A $t$ test for related samples was used to differentiate paired mean TRIMP values. Cohen's $d$ was used for effect size, 0.2 or below was considered as low, between 0.2 and 0.5 as moderate and above 0.5 as large. Bland and Altman plots were used to evaluate agreement between TRIMP methods. Differences between the measurements of ITL by the four methods were plotted in relation to the mean values; $95 \%$ of the differences were expected lie between the two limits of agreement that were the mean difference $\pm 1.96 \mathrm{SD}$ of the differences, expressed as bias \pm random error. Statistical significance was established at $p<0.05$. 


\section{RESULTS AND DISCUSSION Results}

Lineal relationship $(r=0.699, p<0.01)$ between $\mathrm{HR}$ and ETL was observed during field hockey intermittent training (Figure 1). Determination coefficient $R^{2}=0.489$ dictates that $48.9 \%$ of the HR changes is explained by Player's load. Individual subject analyses indicate stronger relationships between these two variables, with a range of $r=0.701-0.933$, $(p<0.01)$. On an individual basis, Player's load explains HR within a 49.1 and $87 \%$ range.

Table 2 shows individual weighting factors. Descriptive data of Player's load, bTRIMP, eTRIMP, iTRIMP and mTRIMP for each player is presented in Table 3. One-way ANOVA showed significant differences $(p<0.01)$ on all measured parameters. Session ETL between subjects showed an $F$ value of 17.38 . ETA effect size indicates that the subject explains $66.6 \%$ of the Player's load differences. Session ITL by the bTRIMP, eTRIMP, iTRIMP and MTRIMP methods showed $F$ values of 8.74, 8.46, 17.7 and 7.94 respectively. ETA effect size indicate that the subject explains $53.5 \%, 52.9 \%, 67 \%$ and $51.7 \%$ of bTRIMP, eTRIMP, iTRIMP and mTRIMP differences respectively. Minutes per session showed an $\mathrm{F}$ value of 3.5. ETA effect size indicates that the subject explains $37.2 \%$ of minute's differences.

Table 4 shows descriptive data for Player's load:minute and TRIMP:minute ratios. Player's load:minute ratio shows an $\mathrm{F}$ value of 9.02 . ETA effect size indicates that the subject explains $54.1 \%$ of the ratio's differences. Differences for TRIMP:minute ratios at bTRIMP, eTRIMP, iTRIMP and MTRIMP show $F$ values of 10.11, 9.68, 19.12 and 10.17 respectively. ETA effect size indicates that the subject explains $56.3 \%, 55.5 \%, 68.4 \%$ and $56.4 \%$ of the TRIMP:minute ratio differences respectively.

Table 5 shows descriptive data for the four TRIMP methods TRIMP:Player's load ratios. Between subjects TRIMP:Player's load ratios for bTRIMP, eTRIMP, iTRIMP and

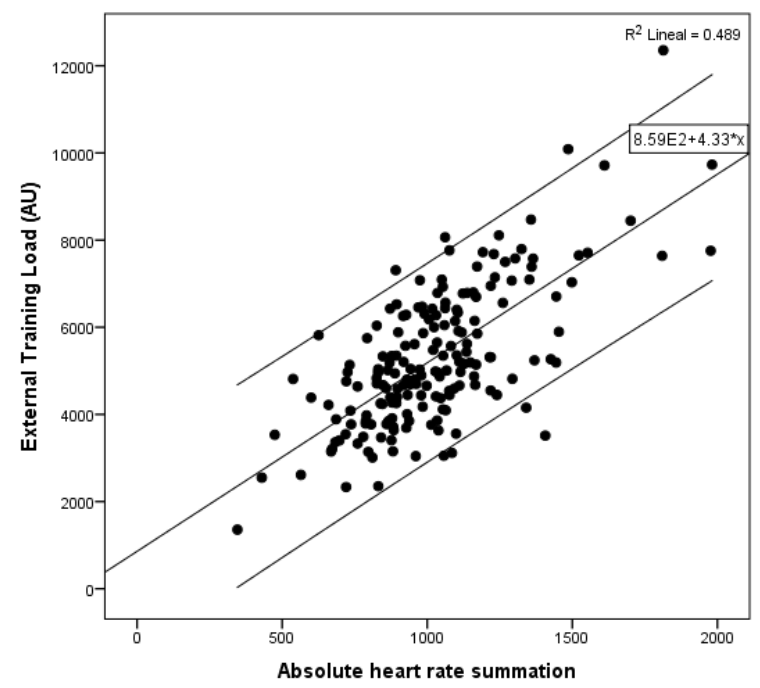

Figure 1. Scatter plot between ETL and HR in beats per minute.

Table 2. Weighting factors according to exponential equation $y=a^{*} e^{b^{*} x}$. Tabla 2. Factores de ponderación de acuerdo a la ecuación exponencial y $=a^{*} e^{b^{*} x}$.

\begin{tabular}{ll}
\hline Subjects' weighting factors & \\
\hline Subject $01=0.33 \mathrm{e}^{3.09 x}$ & Subject $07=0.37 \mathrm{e}^{3.76 x}$ \\
Subject $02=0.21 \mathrm{e}^{4.13 x}$ & Subject $08=0.28 \mathrm{e}^{3.96 x}$ \\
\hline Subject $03=0.54 \mathrm{e}^{3.34 x}$ & Subject $09=0.18 \mathrm{e}^{4.24 x}$ \\
Subject $04=1.26 \mathrm{e}^{2.49 x}$ & Subject $10=0.23 \mathrm{e}^{3.98 x}$ \\
Subject $05=0.12 \mathrm{e}^{4.7 x}$ & Subject $11=0.34 \mathrm{e}^{3.83 x}$ \\
Subject $06=1.20 \mathrm{e}^{2.52 x}$ & Team $=0.42 \mathrm{e}^{3.45 x}$ \\
\hline Note. - $\mathrm{e}=$ Neperian Logarithm 2.7182. &
\end{tabular}

mTRIMP show $F$ values of 4.97, 13.43, 26.15 and 4.61 respectively. ETA effect size indicate that subjects explained $43.1 \%$, $61.7 \%, 73.9 \%$ and $41.8 \%$ of the differences in TRIMP:Player's load ratios respectively.

Large to almost perfect correlation coefficients between the four TRIMP methods show that they follow

Table 3. Session's descriptive values of minutes, "Players load”, eTRIMP, bTRIMP, mTRIMP and iTRIMP of each subject.

Tabla 3. Valores descriptivos de minutos, "Carga del jugador", eTRIMP, bTRIMP, mTRIMP e iTRIMP por sesión de cada sujeto.

\begin{tabular}{|c|c|c|c|c|c|c|c|c|c|c|c|c|}
\hline \multirow[t]{2}{*}{ Subject } & \multicolumn{2}{|c|}{ Minutes } & \multicolumn{2}{|c|}{ ETL } & \multicolumn{2}{|c|}{ bTRIMP } & \multicolumn{2}{|c|}{ eTRIMP } & \multicolumn{2}{|c|}{ mTRIMP } & \multicolumn{2}{|c|}{ iTRIMP } \\
\hline & Mean & SD & Mean & SD & Mean & SD & Mean & SD & Mean & SD & Mean & SD \\
\hline 01 & 148.25 & 29.72 & 6981.06 & 1697.18 & 132.09 & 52.28 & 137.75 & 59.85 & 157.60 & 61.56 & 182.81 & 87.51 \\
\hline 02 & 114.87 & 30.04 & 5828.90 & 1204.89 & 95.75 & 30.43 & 117.20 & 35.40 & 122.38 & 38.61 & 192.22 & 74.18 \\
\hline 03 & 141.08 & 29.43 & 4265.52 & 1089.63 & 109.12 & 36.57 & 127.68 & 41.26 & 122.38 & 40.28 & 251.63 & 100.02 \\
\hline 04 & 123.75 & 24.34 & 4705.05 & 740.76 & 86.98 & 28.69 & 141.25 & 42.70 & 111.39 & 37.01 & 315.66 & 113.56 \\
\hline 05 & 138.76 & 29.10 & 4018.48 & 954.27 & 59.56 & 23.86 & 65.08 & 26.05 & 70.80 & 29.79 & 60.59 & 40.07 \\
\hline 06 & 137.62 & 30.15 & 5188.07 & 1169.93 & 135.54 & 44.35 & 160.95 & 54.22 & 152.71 & 50.77 & 355.26 & 123.67 \\
\hline 07 & 134.54 & 18.63 & 5234.46 & 1305.02 & 86.07 & 22.86 & 120.77 & 33.99 & 102.89 & 26.77 & 182.61 & 67.22 \\
\hline 08 & 143.26 & 38.63 & 6449.14 & 1317.20 & 123.54 & 37.65 & 140.61 & 43.20 & 139.76 & 41.43 & 223.83 & 86.72 \\
\hline 09 & 139.24 & 26.54 & 6029.32 & 1816.04 & 129.48 & 50.99 & 149.12 & 65.89 & 150.49 & 59.59 & 209.59 & 120.87 \\
\hline 10 & 123.24 & 31.74 & 4802.38 & 1407.32 & 87.79 & 51.91 & 122.29 & 69.63 & 104.81 & 58.97 & 145.10 & 105.77 \\
\hline 11 & 158.91 & 35.72 & 7696.08 & 1909.43 & 90.74 & 26.34 & 88.88 & 23.40 & 104.21 & 28.22 & 153.03 & 50.40 \\
\hline
\end{tabular}

$\mathrm{SD}=$ Standard deviation 


\section{González-Fimbres et al: Biotecnia / XXI (3): 26-34 (2019)}

Table 4. All session's descriptive values for "Players load"/min, bTRIMP/min, eTRIMP/min, $\mathrm{mTRIMP} / \mathrm{min}$, and iTRIMP/min.

Tabla 4. Valores descriptivos de los índices de "Carga del jugador"/min, bTRIMP/min, eTRIMP/min, mTRIMP/min e iTRIMP/min de todas las sesiones de entrenamiento.

\begin{tabular}{|c|c|c|c|c|c|c|c|c|c|c|}
\hline \multirow[b]{2}{*}{ Subject } & \multicolumn{2}{|c|}{ Player's load/min } & \multicolumn{2}{|c|}{ bTRIMP/min } & \multicolumn{2}{|c|}{ eTRIMP/min } & \multicolumn{2}{|c|}{ mTRIMP/min } & \multicolumn{2}{|c|}{ iTRIMP/min } \\
\hline & Mean & SD & Mean & SD & Mean & SD & Mean & SD & Mean & SD \\
\hline 01 & 47.44 & 7.57 & 0.90 & 0.29 & 0.95 & 0.38 & 1.07 & 0.35 & 1.25 & 0.51 \\
\hline 02 & 51.88 & 8.09 & 0.84 & 0.22 & 1.04 & 0.28 & 1.07 & 0.26 & 1.67 & 0.57 \\
\hline 03 & 31.00 & 7.73 & 0.79 & 0.26 & 0.94 & 0.34 & 0.89 & 0.29 & 1.85 & 0.78 \\
\hline 04 & 38.59 & 5.10 & 0.70 & 0.18 & 1.15 & 0.29 & 0.90 & 0.22 & 2.53 & 0.70 \\
\hline 05 & 29.30 & 6.07 & 0.43 & 0.14 & 0.47 & 0.17 & 0.51 & 0.18 & 0.43 & 0.26 \\
\hline 06 & 38.11 & 6.35 & 0.99 & 0.26 & 1.18 & 0.36 & 1.12 & 0.30 & 2.60 & 0.76 \\
\hline 07 & 39.28 & 9.13 & 0.65 & 0.17 & 0.91 & 0.26 & 0.77 & 0.20 & 1.37 & 0.52 \\
\hline 08 & 47.23 & 13.85 & 0.91 & 0.39 & 1.05 & 0.52 & 1.03 & 0.40 & 1.70 & 1.08 \\
\hline 09 & 43.43 & 9.85 & 0.93 & 0.28 & 1.07 & 0.39 & 1.08 & 0.33 & 1.49 & 0.70 \\
\hline 10 & 39.65 & 9.77 & 0.71 & 0.36 & 1.01 & 0.54 & 0.86 & 0.42 & 1.19 & 0.81 \\
\hline 11 & 52.74 & 25.20 & 0.58 & 0.14 & 0.59 & 0.20 & 0.67 & 0.16 & 1.00 & 0.36 \\
\hline
\end{tabular}

$\mathrm{SD}=$ Standard deviation

Table 5. All training session's adjusted (multiplied by 100 for a better management) descriptive values of bTRIMP, eTRIMP, mTRIMP e iTRIMP and "Players load" indexes.

Tabla 5. Valores descriptivos de los índices de bTRIMP, eTRIMP, mTRIMP e iTRIMP y "Carga del jugador" ajustados (multiplicados por 100 para un mejor manejo) de todas las sesiones de entrenamiento.

\begin{tabular}{|c|c|c|c|c|c|c|c|c|}
\hline \multirow[t]{2}{*}{ Subject } & \multicolumn{2}{|c|}{ bTRIMP } & \multicolumn{2}{|c|}{ eTRIMP } & \multicolumn{2}{|c|}{ mTRIMP } & \multicolumn{2}{|c|}{ iTRIMP } \\
\hline & Mean & SD & Mean & SD & Mean & SD & Mean & SD \\
\hline 01 & 1.86 & 0.40 & 1.95 & 0.54 & 2.22 & 0.46 & 2.55 & 0.76 \\
\hline 02 & 1.62 & 0.31 & 1.99 & 0.38 & 2.07 & 0.39 & 3.22 & 0.89 \\
\hline 03 & 2.56 & 0.58 & 3.02 & 0.74 & 2.88 & 0.66 & 5.87 & 1.77 \\
\hline 04 & 1.83 & 0.49 & 2.99 & 0.72 & 2.35 & 0.60 & 6.64 & 1.89 \\
\hline 05 & 1.46 & 0.37 & 1.60 & 0.43 & 1.73 & 0.46 & 1.43 & 0.68 \\
\hline 06 & 2.57 & 0.41 & 3.05 & 0.58 & 2.89 & 0.46 & 6.71 & 1.20 \\
\hline 07 & 1.71 & 0.50 & 2.39 & 0.71 & 2.04 & 0.57 & 3.58 & 1.30 \\
\hline 08 & 1.91 & 0.39 & 2.18 & 0.51 & 2.16 & 0.43 & 3.46 & 1.13 \\
\hline 09 & 2.11 & 0.30 & 2.41 & 0.47 & 2.45 & 0.35 & 3.28 & 1.04 \\
\hline 10 & 1.73 & 0.65 & 2.44 & 0.95 & 2.08 & 0.75 & 2.81 & 1.57 \\
\hline 11 & 1.45 & 1.89 & 1.34 & 1.32 & 1.65 & 2.04 & 2.39 & 2.87 \\
\hline
\end{tabular}

$\mathrm{SD}=$ Standard deviation .

the same tendencies (Table 6). Effect size between TRIMP methods correlations were from medium to large, at the $\mathrm{R}^{2}=$ 0.652 to 0.968 range.

Mean values of bTRIMP, eTRIMP, iTRIMP, and mTRIMP were $102.76 \pm 44.16,121.44 \pm 53.36,201.03 \pm 118.33$, and $120.33 \pm 50.33$ respectively. Differences were observed between bTRIMP and eTRIMP $\left(t_{(228)}=13.9, p<0.01\right)$, mTRIMP $\left(t_{(228)}=-28.7, p<0.01\right)$ and $\operatorname{iTRIMP}\left(t_{(228)}=-17.4, p<0.01\right)$. Differences were also found between iTRIMP and bTRIMP $\left(t_{(228)}\right.$ $=17.4, p<0.01)$, eTRIMP $\left(t_{(228)}=-16.3, p<0.01\right)$ and mTRIMP $\left(t_{(228)}=-15.09, p<0.01\right)$. There were no differences between eTRIMP and mTRIMP (Figure 2). Effect size was moderate between bTRIMP with eTRIMP $(d=0.38)$ and $\operatorname{mTRIMP}(d=0.37)$;
Table 6. Correlation coefficients between all four TRIMP methods. Tabla 6. Coeficientes de correlación entre los cuatro métodos de TRIMP.

\begin{tabular}{llccc}
\hline & $\mathbf{1}$ & $\mathbf{2}$ & $\mathbf{3}$ \\
\hline 1 & eTRIMP & - & & \\
2 & bTRIMP & $0.913^{* *}$ & - & \\
3 & mTRIMP & $0.932^{* *}$ & $0.984^{* *}$ & - \\
4 & iTRIMP & $0.893^{* *}$ & $0.808^{* * *}$ & $0.816^{* *}$ \\
\hline
\end{tabular}

${ }^{* *}$ Correlation coefficient to $p<0.01$.

and large with iTRIMP $(d=1.10)$. Large effects sizes were found between iTRIMP and eTRIMP $(d=0.86)$ and MTRIMP $(d=0.88)$. Since no differences were found between eTRIMP and mTRIMP, level of agreement was assessed using the

\section{Volumen XXI, Número 3}




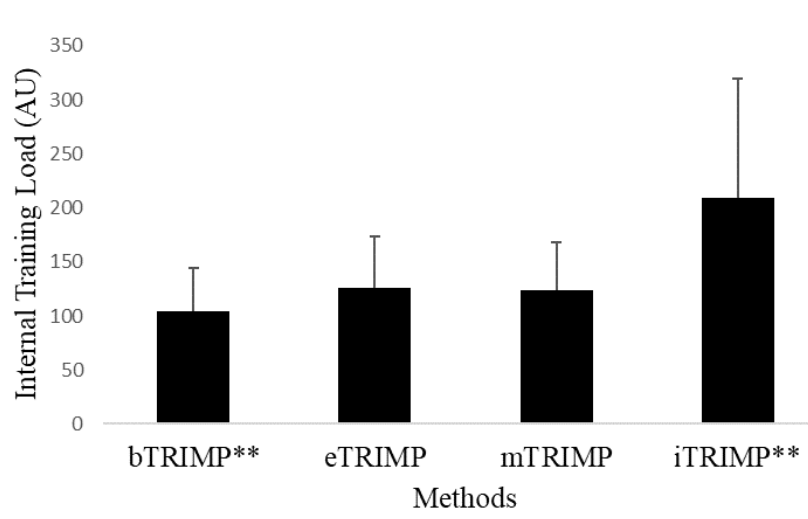

Figure 2. Comparison between mean values of ITL in Arbitrary Units (AU) between all four TRIMP methods. **Differences with other methods at $p<$ 0.01 .

Bland and Altman Plot (Figure 3). Lineal regression analysis between paired measurements differences and means show high significance of $t(p<0.01)$, which indicates bias between methods and a lack of agreement.

\section{ETRIMP vs MTRIMP}

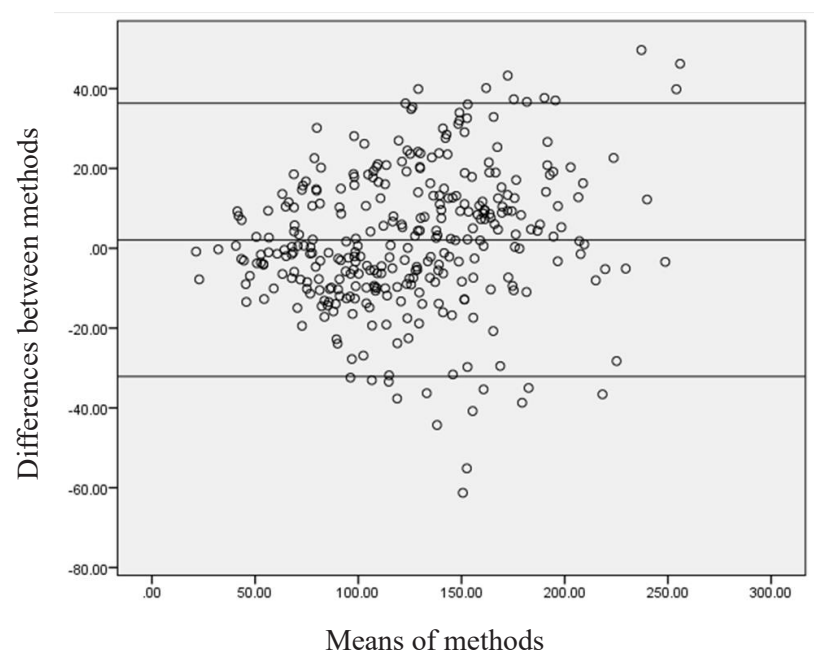

Figure 3. Bland and Altman Plot between eTRIMP and mTRIMP methods. Centerline represents the mean of the differences between methods, superior and inferior lines represent confidence intervals at $96 \%$.

There was significant correlation between TRIMP and Player's load $(p<0.01)$. Large correlation was found between Player's load with bTRIMP $\left(r=0.509, \mathrm{R}^{2}=0.259\right)$ and MTRIMP ( $\left.r=0.516, \mathrm{R}^{2}=0.267\right)$. Moderate correlation was found with eTRIMP $\left(r=0.336, \mathrm{R}^{2}=0.113\right)$. Small correlation was found with iTRIMP ( $\left.r=0.224, \mathrm{R}^{2}=0.050\right)$. Effect sizes range from small to medium as shown in Figure 4.

\section{Discussion}

Due to the belief of HR's delayed kinetics during highintensity exercise, and the assumption that it does not respond well to maximal anaerobic efforts, the use of HR-based TRIMP methods for ITL quantification at intermittent sports has been questioned (Buchheit et al., 2013; García-Ramos et
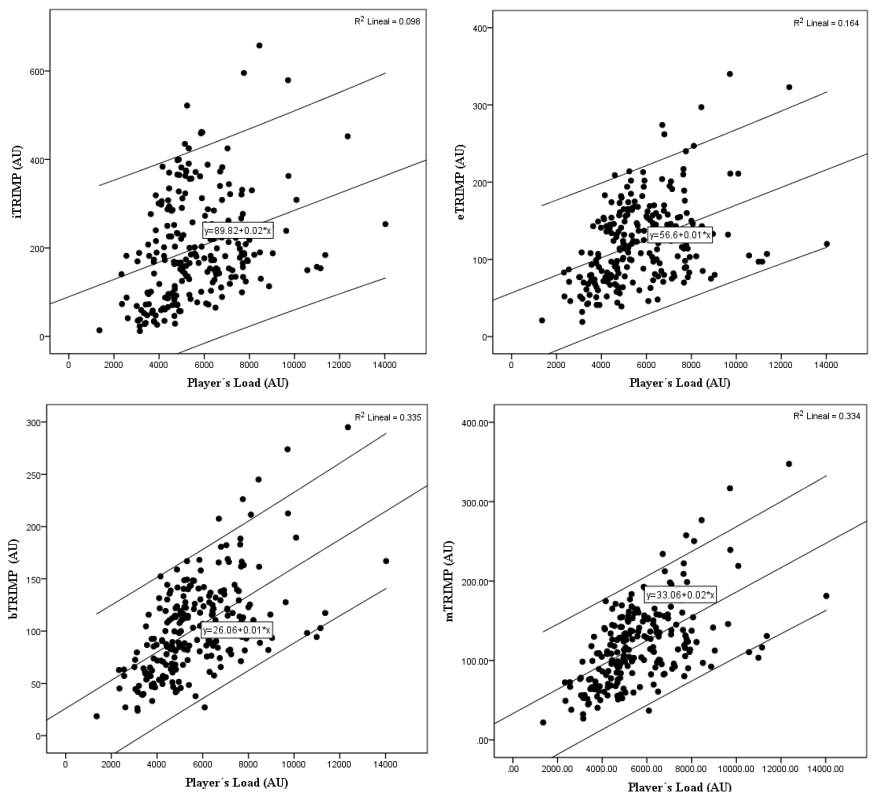

Figure 4. Scatter plots and determination coefficient between TRIMP methods and "Players load".

al., 2014; Saboul et al., 2015). Nevertheless, we found a significant relationship between absolute HR and Player's load in this study. It was found that HR followed a similar behavior than Player's load during intermittent training sessions, verifying reports pointing to a lineal relationship between HR and ETL intensity (Lucia et al., 2003). When relationships are analyzed on an individual basis, even greater correlation coefficients were found in contrast to group analysis. Proving that HR responses to ETL depend on individual characteristics, conditioning level in particular (Manzi et al., 2009). This relationship indicates that $\mathrm{HR}$ responses during field hockey training accurately represent players' external effort.

The most recent TRIMP method proposed by Manzi (2009) sustain that Bla curve plotted against fractional elevation in HR shifts to the right with performance improvement (Manzi et al., 2009; Manzi et al., 2010; Manzi et al., 2013; Manzi et al., 2015). As fractional HR (HRres) takes into account HRmin and HRmax for its calculation, one may think that it has an element for the subject's individual capacity, and in that regard, it is supposed to reflect individual internal exercise intensity. Nevertheless, results show different individual weighting factors, as curves show distinctive subject's Bla responses at the same HRres ranges. This affirms the idea first declared by Stagno et al. (2007) and later by Manzi et al. (2009; 2010). This gives us a basis to think that HRres alone is not an accurate marker for internal exercise intensity, and that coaches should consider Bla responses for HR assessment in training.

Some studies compared ETL differences between various exercise modes (Montgomery et al., 2010; Weaving et al., 2017), match periods and high and low success teams (Hulin et al., 2014), training and competition (Delaney et al., 2016), and ITL values (Casamichana et al., 2013; Scanlan et al., 2014). These studies compared group mean values, but as far 
as we know, there are not studies that analyzed individual ETL differences between subjects submitted to the same training sessions. Data in this study show disparities in Player's load values between subjects. This suggests that players are not exposed to the same external physical demands during training sessions, which opens up a new line of analysis in function of knowing if ETL differences have a positive or negative impact on the magnitude of the stimulus required to incite training adaptation.

Previous studies (Borresen \& Lambert, 2008; Borresen \& Lambert, 2009; Lambert \& Borresen, 2010; Mujika, 2017) affirm that the same ETL can elicit differentiated internal responses according to individual characteristics and conditioning levels. This is confirmed in this study, observing different subject's ITL mean values, even if all of them attended the same training sessions. Data suggests that large effect sizes in ITL differences are partially explained by differences in relative and absolute ETL values between subjects. Also, this could be affected by different playing positions, albeit a positional analysis was not made in this study, previous studies have reported that playing position has a significant effect in physiological responses (Buglione et al., 2013). Another explanation can be the differences observed in aerobic capacity expressed as $\mathrm{VO}_{2}$ max, which was calculated by field tests (Buchheit, 2010) prior to protocol beginning.

Lower values in comparison to the other three methods suggest that bTRIMP underestimates training stimulus. This can be explained by the use of session's mean HRres (Banister, 1991), which equals continuous training at medium intensity to intermittent training incorporating high and low intensities when both of them have the same mean HRres (Saboul et al., 2015). As mTRIMP consider each $H R$ value, it weights in high intensity efforts, emitting higher values, even if both methods use the same weighting factor. Contrasting mTRIMP and bTRIMP, verifies that bTRIMP underestimates ITL, in particular during interval and intermittent training. ETRIMP classify HR values in training zones by HRmax percentage (Edwards, 1994). Even if there were no differences in mean values between eTRIMP and MTRIMP, Bland and Altman Plot show that there is no agreement between them. This seems to imply that even if using training zones is more appropriate than averaging HRres, considering every $H R$ value prevents over- or underestimation of ITL when HR values are near to eTRIMP zone limits. TRIMP methods using generic weighting factors (bTRIMP, eTRIMP and mTRIMP) did not show great inter-subject variability, evidenced by lower values of SD. This agrees with another team sport study (Akubat et al., 2012), which reported a high SD variability in ITRIMP in soccer players. This supports that iTRIMP is more sensitive to individual internal responses to ETL. For this reason, iTRIMP is considered a suitable method for inter-subject ITL assessment in intermittent team sports as previously recommended (Akubat et al., 2012; Castagna et al., 2013; Akubat et al., 2014; Malone \& Collins, 2016).
Despite differences in mean values, high correlation between TRIMP methods indicates they may have similar responses to ETL. This suggests that any of them can be considered as a useful tool for ITL assessment given that results are emitted in arbitrary units and assuming an inter-subject analysis is not necessary.

Scanlan et al. (2014) established the relationship between bTRIMP and eTRIMP with Player's load. While no reference for iTRIMP/Player's load relationship was found, a lower correlation coefficient with the rest of the methods was observed. This was an expected result, given that all subjects were included in the sample. Extremely diverse weighting factors incite differentiated ETL responses for each subject, which result in a lower general correlation coefficient. Contrary to the rest of the methods, which having the same weighting factor for every subject elicit consistent relationships.

\section{CONCLUSSIONS}

HR responses during intermittent sports training, like field hockey, are tightly related to TL in high and low intensity stimulus, which allows to use HR based TRIMP methods for quantification and assessment of ITL. bTRIMP is considered to underestimate internal responses to ETL. ETRIMP and the new MTRIMP more precisely represent general internal responses compared to bTRIMP. Nevertheless, iTRIMP better reflects individual responses to ETL. Even if is highly recommendable to use iTIMP to ITL quantification, MTRIMP can be a valid option when coaches do not have access to perform incremental effort tests for Bla profile determination. A combination of ITL and ETL methods must be used for a global asessment of trainig loads.

\section{ACKNOWLEDGMENTS}

We would like to thank Sonora State Sports Commission (Comisión del Deporte del Estado de Sonora, CODESON) for allowing us to carry out this study, to the Sonora's field hockey team coaching staff, as well as the players who volunteered for this study.

\section{REFERENCES}

Abdelkrim, N. Ben, Fazaa, S. El., \& Ati, J. El. 2007. Time-motion analysis and physiological data of elite under-19 year old basketball players during competition. British journal of sports medicine. 41(2): 69-75.

Akubat, I., Patel, E., Barrett, S., \& Abt, G. 2012. Methods of monitoring the training and match load and their relationship to changes in fitness in professional youth soccer players. Journal of Sports Science and Medicine. 30(14): 1473-1480.

Akubat, I., Barrett, S. \& Abt, G. 2014. Integrating the Internal and External Training Load in Soccer. International Journal of Sport Physiology and Performance. 9(3): 457-462.

Alexiou, H. \& Coutts, A.J. 2008. A comparison of methods used for quantifying internal training load in women soccer players. International Journal of Sports Physiology and Performance. 3(3): 320-330. 
Banister, E.W. 1991. Modeling Elite Athletic Performance 2nd ed. J. D. MacDougall, H. A. Wenger, \& H. J. Green, eds., Champaign, Illinois: Human Kinetics.

Borresen, J. \& Lambert, M.I. 2008. Quantifying Training Load: A Comparison of Subjective and Objective Methods. International Journal of Sports Physiology and Performance. $3(1): 16-30$.

Borresen, J. \& Lambert, M.I. 2009. The Quantification of Training Load , the Training Response and the Effect on Performance. Sports Medicine. 39(9): 779-795.

Buchheit, M. et al. 2013. Monitoring fitness, fatigue and running performance during a pre-season training camp in elite football players. Journal of Science and Medicine in Sport. 16(6): 550-555.

Buchheit, M. 2010. The 30-15 Intermittent Fitness Test : 10 year review. Myorobie Journal. 1:1-9.

Buchheit, M. 2014. Monitoring training status with HR measures : Do all roads lead to Rome? Frontiers in Physiology, 5 (73):1-19 . http://doi.org/10.3389/fphys.2014.00073

Buglione, A. et al. 2013. Physical and Physiological demands of elite and sub-elite Field Hockey players. International Journal of Performance in Sport. 13(3): 872-884.

Canovas, M. et al. 2014. Time-Motion Analysis Procedure in Team Sports: Example for Youth Basketball. Strength \& Conditioning Journal. 36(3): 71-75.

Casamichana, D. et al. 2013. Relationship between indicators of training load in soccer players. The Journal of Strength \& Conditioning Research. 27(2): 369-374.

Castagna, C. et al. 2013. Pre-Season Variations in Aerobic Fitness and Performance in Elite Standard Soccer Players: a TeamStudy. Journal of strength and conditioning research. 27(11): 2959-2965.

Delaney, J., Duthie, G.M., Thornton, H.R., Scott, T.J., Gay, D., \& Dascombe, B.J. 2016. Acceleration-Based Running Intensities of Professional Rugby League Match-Play. International Journal of Sport Physiology and Performance. 11(6): 802-809.

Deweese, B.H., Hornsby, G., Stone, M., Stone, M.H. 2015. The training process : Planning for strength - power training in track and field . Part $1:$ Theoretical aspects. Journal of Sport and Health Science. 4(4): 308-317.

Edwards, S. 1994. The heart rate monitor book. Medicine \& Science in Sports \& Exercise. 26(5): 647.

Fitz-Clarke, J.R., Morton, R.H., \& Banister, E.W. 1991. Optimizing athletic performance by influence curves. Journal of Applied Physiology. 71(3): 1151-1158.

García-ramos, A., Feriche, B., Calderón, C., Iglesias, X., Barrero, A., Chaverri, D., Schuller, T., \& Rodríguez, F.A. 2014. Training load quantification in elite swimmers using a modified version of the training impulse method. European Journal of Sport Science. 15(2): 85-93.

Halson, S.L. 2014. Monitoring Training Load to Understand Fatigue in Athletes. Sports medicine. 44(2): 139-147.

Hulin, B., Gabbett, T.J., Kearney, S., \& Corvo, A. 2015. Physical demands of match-play in successful and less-successful elite rugby league teams. International Journal of Sports Physiology and Performance. 10(6): 703-710.

Jennings, D.H., Cormack, S.J., Coutts, A.J., \& Aughey, R.J. 2012. International Field Hockey Players Perform More HighSpeed Running Than National-Level Counterparts. Journal of strength and conditioning research. 26(4): 947-952.
Klusemann, M.J., Pyne, D.B., Hopkins, W.G., \& Drinkwater, E.J. 2013. Activity profiles and demands of seasonal and tournament basketball competition. International Journal of Sport Physiology and Performance. 8(6): 623-629.

Lambert, M.I., \& Borresen, J. 2010. Measuring Training Load in Sports. International Journal of Sports Physiology and Performance. 5(3): 406-411.

Lucia, A., Hoyos, J., Santalla, A., Earnest, C., \& Chicharro, J.L. 2003. Tour de France versus Vuelta a España: which is harder? Medicine and Science in Sports and Exercise. 35(5): 872-878.

Malone, S., \& Collins, K. 2016. Relationship between individualised training impulse and aerobic fitness measures in Hurling players across a training period. Journal of Strength and Conditioning Research. 30(11): 3140-3145.

Manzi, V., Castagna, C., Padua, E., Lombardo, M., D'Ottavio, S., Massaro, M., Volterrani, M., \& lellamo, F. 2009. Dose-response relationship of autonomic nervous system responses to individualized training impulse in marathon runners. American Journal of Physiology. Heart and Circulatory Physiology. 296(6): 1733-1740.

Manzi, V., Bovenzi, A., Impellizzeri, F.M., Carminati, I., \& Castagna, C. 2013. Individual Training-Load and Aerobic-Fitness Variables in Premiership Soccer Players During the Precompetitive Season. Journal of strength and conditioning research. 27(3): 631-636.

Manzi, V., D'Ottavio, S., Impellizzeri, F.M., Chaouachi, A., Chamari, K., \& Castagna, C. 2010. Profile of Weekly Training Load in Elite Male Professional Basketball Players. Journal of strength and conditioning research. 24(5): 1399-1406.

Manzi, V., lellamo, F., Impellizzeri, M.F., D'Ottavio, S., \& Castagna, C. 2009. Relation between Individualized Training Impulses and Performance in Distance Runners. Medicine and Science in Sports and Exercise. 41(11): 2090-2096.

Montgomery, P.G., Pyne, D.B., \& Minahan, C.L. 2010. The Physical and Physiological Demands of Basketball Training and Competition. International journal of sports physiology and performance. 5(1): 75-86.

Morton, R.H., Fitz-clarke, J.R. \& Banister, E.W. 1990. Modeling human performance in running. Journal of Applied Physiology. 69(3): 1171-1177.

Mujika, I. 2017. Quantification of Training and Competition Loads in Endurance Sports: Methods and Applications. International Journal of Sports Physiology and Performance. 12(supp.2): 9-17.

Mujika, I. 2013. The alphabet of sport science research starts with Q. International Journal of Sports Physiology and Performance. 8(5): 465-466.

Saboul, D., Balducci, P., Millet, G., Pialoux, V., \& Hautier, C. 2015. A pilot study on quantification of training load: The use of HRV in training practice. European Journal of Sport Science. 16(2): 172-181.

Scanlan, A., Wen, N., Tucker, P.S., \& Dalbo, V.J. 2014. The relationship between internal and external training load models during basketball training. Journal of Strength \& Conditioning Research. 28(9): 2397-2405.

Scott, B.R., Lockie, R.G., Knight, T.J., Clark, A.C., \& Janse de Jonge, X.A. 2013. A Comparison of Methods to Quantify the In-Season Training Load of Professional Soccer Players. International journal of sports physiology and performance. 8(2): 195-202. 
Scott, T., Black, C.R., Quinn, J., \& Coutts, A.J. 2012. Validity and Reliability of the Session-RPE Method for Quantifying Training in Australian Football: A Comparison of the CR10 and CR100 Scales. Journal of Strength \& Conditioning Research. 27(1): 270-276.

Stagno, K., Thatcher, R., \& Van Someren, K. 2007. A modified TRIMP to quantify the in-season training load of team sport players. Journal of Sports Sciences. 25(6): 629-634.

Suarez-Arrones, L., Portillo, J., González-Ravé, J.M., Muñoz, V.E., \& Sanchez, F. 2012. Match running performance in Spanish elite male rugby union using global positioning system. Isokinetics and Exercise Science. 20(2): 77-83.

Turner, A. 2011. The Science and Practice of Periodization: A Brief Review. Strength \& Conditioning Journal. 33(1): 34-46.
Varley, M., Fairweather, I., \& Aughey, R.J. 2012. Validity and reliability of GPS for measuring instantaneous velocity during acceleration, deceleration, and constant motion. Journal of Sports Sciences. 30(2): 121-127.

Vinson, D., \& Peters, D.M. 2016. Position-specific performance indicators that discriminate between successful and unsuccessful teams in elite women's indoor field hockey: implications for coaching implications for coaching. Journal of Sports Sciences. 34(4): 311-320.

Weaving, D., Jones, B., Till, K., Marshall, P., Earle, K., \& Abt, G. 2017. Quantifying The External And Internal Loads Of Professional Rugby League Training Modes: Consideration for Concurrent Field-Based Training Prescription. Journal of Strength and Conditioning Research. 11: 1-23. 